\title{
A FADIGA NOS PACIENTES COM CÂNCER DE LARINGE
}

\author{
Fernanda Mara Ribeiro Coelho* \\ Namie Okino Sawada**
}

COELHO, F.M.R.; SAWADA, N.O. A fadiga nos pacientes com câncer de laringe. Rev.latino-am.enfermagem, Ribeirão Preto, v. 7, n. 5, p. 103-107, dezembro 1999.

Este estudo levantou a presença de sintomas subjetivos de fadiga nos pacientes laringectomizados. Utilizamos o instrumento proposto por YOSHITAKE ${ }^{27}$ que investiga os sintomas de mal-estar, cansaço mental e sensações especificas de cansaço. Os dados foram confrontados com a literatura e demonstraram que os fatores psicológicos, o tratamento e a fase da doença estão diretamente relacionados.

UNITERMOS: fadiga, pacientes, neoplasias laringeas

\section{INTRODUÇÃO}

Fadiga é uma experiência universal que geralmente desaparece após uma boa noite de repouso, mas em muitos pacientes oncológicos esta sensação persiste, torna-se crônica, desagradável e de exaustão contínua $^{14}$.

De acordo com RHODES et al. ${ }^{18}$ a fadiga apresentada por indivíduos com doenças crônicas é diferente da fadiga diária, este tipo se apresenta como um sintoma complexo provocado por condições físicas e psicológicas preexistentes. Em muitas doenças crônicas incluindo o câncer, a fadiga é um sintoma comumente angustiante.

Algumas pesquisas evidenciaram que uma alta porcentagem de pacientes com câncer sofrem de fadiga, este sintoma é exacerbado pelo tratamento como radioterapia e quimioterapia e com Interferon Alpha $2^{4,7,13}$.

Segundo MARZIALE ${ }^{10}$, apesar da fadiga ser um fenômeno preocupante desde o início dos estudos das relações do trabalho, é a partir da década de 20 que as tentativas de conceituar fadiga se tornam mais marcantes. Simultaneamente neste período, são encontrados relatos quanto às dificuldades de aferição e interpretação por se tratar de um fenômeno bastante complexo ${ }^{10}$.

POTEMPA $^{17}$ relata que fadiga é uma queixa comum e em muitos casos está relacionada com eventos específicos como pouco sono ou falta de exercícios, nesses casos a fadiga é breve e facilmente remediável. Entretanto, quando ela persiste por muito tempo, tornase debilitante interferindo no cotidiano do indivíduo. Essa condição é freqüentemente referida como fadiga crônica e é encontrada em grande porcentagem da população com problemas crônicos de saúde.

RYAN $^{19}$ define a fadiga como um estado geral do indivíduo, resultante da atividade contínua de trabalho.

Fadiga, para MAYO ${ }^{11}$, não é uma entidade, e sim uma palavra incômoda para descrever uma grande variedade de fenômenos.

De acordo com CAMERON ${ }^{2}$, fadiga é uma resposta generalizada ao estresse sobre um período de tempo com efeitos que podem ser agudos ou crônicos, confinados ao estado subjetivo ou estendido dentro dos aspectos mensuráveis do desempenho do indivíduo. Coloca ainda em evidência a questão da importância de variáveis, tais como ansiedade e distúrbios do sono, que influenciam diretamente no aparecimento da fadiga.

VOLLE et al. ${ }^{24}$ considera fadiga como um estado global proveniente do desequilíbrio interno principalmente no sistema de relações do organismo.

No entender de LINO $^{9}$ a fadiga revela uma alteração reversível da coordenação das funções biológicas, físicas e psíquicas, pois, se retiradas as causas que a provocam, coloca novamente o organismo em situação normal. No entanto, se persistirem ou se agravarem, tornam-se patológicas.

Encontramos no dicionário da língua portuguesa

\footnotetext{
* Aluna do $4^{\circ}$ Semestre de Graduação da Escola de Enfermagem de Ribeirão Preto da Universidade de São Paulo e bolsista do Programa Especial de Treinamento PET/CAPES

** Professor Doutor do Departamento de Enfermagem Geral e Especializada da Escola de Enfermagem de Ribeirão Preto da Universidade de São Paulo
} 
de FERREIRA ${ }^{5}$, como sinônimos de fadiga: cansaço, canseira, fadigamento, diminuição gradual da resistência de um material por efeito de solicitações repetidas.

A fadiga, na perspectiva da enfermagem, pode ser definida como uma sensação subjetiva de exaustão influenciada pelo ritmo circadiano, podendo variar quanto a sua duração, freqüência e grau ${ }^{14}$.

Assim como existem vários conceitos em torno da fadiga, existem também vários sistemas de classificação, mas muitos deles são de pouca validade para a enfermagem. Porém, o conceito de fadiga crônica e aguda, que caracteriza o sintoma de acordo com sua duração, é de alguma valia. Segundo PIPER ${ }^{14}$, na forma aguda, a fadiga é um mecanismo protetor, mas quando se torna excessiva ou constante (crônica) deixa de ser um mecanismo protetor e pode levar a uma fuga de atividade.

Os indicadores da fadiga, citados na literatura, podem ser objetivos como os fisiológicos, bioquímicos e comportamentais, e subjetivos que são indicadores percebidos como sentimentos e sensações específicas de cansaço $^{20,25,27}$.

Vários estudos com trabalhadores comprovaram a validade da lista de cansaço que compreende um instrumento de trinta sintomas de cansaço, onde o respondente assinala a presença ou não. Este instrumento apresenta 3 subgrupos de sintomas de mal-estar, relacionados a sentimentos gerais de incongruidade, cansaço mental e sensações específicas de cansaço ${ }^{26}$. Acreditamos que esse instrumento é adequado para verificar a presença de fadiga nos pacientes laringectomizados totais.

Diante do exposto, este trabalho propõe-se a levantar dados sobre a fadiga em pacientes oncológicos, especificamente os laringectomizados, que após a cirurgia tem o aparelho respiratório alterado. Analisaremos estes dados a partir da classificação de fadiga aguda ou crônica e seus indicadores subjetivos. Utilizaremos o instrumento proposto por YOSHITAKE ${ }^{26}$.

Assim, o objetivo deste trabalho é detectar através de indicadores subjetivos, sintomas de fadiga nos pacientes laringectomizados.

\section{METODOLOGIA}

Realizamos uma pesquisa não-experimental do tipo survey. Onde foram levantados os sintomas de fadiga nos pacientes laringectomizados.

A coleta de dados foi realizada através de um questionário auto-administrado com questões fechadas e semi-abertas, onde os pacientes laringectomizados assinalaram os sintomas subjetivos de fadiga que no momento estão apresentando (Anexo 1).
Antes de responder ao questionário os pacientes foram instruídos quanto ao preenchimento do mesmo e solicitado o consentimento informado referente a sua participação na pesquisa.

O período de coleta de dados foi de três meses consecutivos. A amostra foi constituída de todos os pacientes, que sabiam ler e escrever e concordaram em participar da pesquisa, que fizeram parte das reuniões do GARPO-laringectomizados (Grupo de apoio e reabilitação da pessoa ostomizada - laringectomizados) no período da coleta de dados.

As reuniões do GARPO ocorrem todas as últimas quartas-feiras do mês e participam desse grupo pacientes que foram submetidos à larigectomia total, familiares e uma equipe multiprofissional.

Para a análise dos dados quantitativos utilizamos os programas FoxPro 2.0 e Epi-Info.

\section{RESULTADOS}

Foram coletados, nas reuniões do GARPO, o total de onze questionários de acordo com a metodologia proposta no período de três meses consecutivos, sendo que 11 pacientes $(100 \%)$ eram do sexo masculino; 6 $(54,5 \%)$ na faixa etária entre 50 e 60 anos, $4(36,4 \%)$ entre 40 e 50 anos e $1(9,1 \%)$ entre 60 e 70 anos.

Esses dados corroboram com o levantamento estatístico de BRASIL, MS. ${ }^{1}$ que aponta a incidência do câncer de laringe maior nos homens do que nas mulheres e predominantemente na faixa etária de 50 anos.

Com relação ao intervalo do início da doença e a primeira cirurgia encontramos 3 pacientes $(27,3 \%)$ entre 3 e 6 meses, $3(27,3 \%)$ entre 6 e 9 meses, $2(18,2 \%)$ não souberam informar e $1(9,1 \%)$ entre 9 e 12 meses.

Dos 11 pacientes estudados $6(54,5 \%)$ fizeram tratamento com radioterapia, $1(9,1 \%)$ com quimioterapia e $4(36,4 \%)$ fazem uso de medicamento.

HAYLOCK \& $\mathrm{HART}^{7}$ relataram que em 30 pacientes adultos com câncer submetidos a diagnostico e tratamento, apresentaram fadiga durante o tratamento com radiação e diminuíram os sintomas após o término deste. Em contraste a esses autores o estudo de NAIL ${ }^{13}$ encontrou aumento da fadiga física após o término do tratamento com radiação em mulheres submetidas à tratamento do câncer ginecológico.

Vários estudos tem associado a fadiga com o tratamento radioterápico em pacientes com câncer, mas essa relação ainda necessita ser investigada.

Ao investigarmos os sintomas gerais de mal-estar 5 pacientes $(45,5 \%)$ apresentaram sensação de cansaço no corpo todo; $4(36,4 \%)$ apresentaram sensação de cansaço nas pernas, cansaço nos olhos, movimentos 
tornam-se duros e desajeitados, sensação de instabilidade ao ficar parado; $2(18,2 \%)$ relataram sensação de cérebro quente e $1(9,1 \%)$ referiu torpor e desejo de se deitar.

No item cansaço mental identificamos 9 pacientes $(81,8 \%)$ que referiram nervosismo; $4(36,4 \%)$ cansaço ao falar, esquecimento fácil, ansiedade constante, pouca paciência e $3(27,3 \%)$ incapacidade de concentração.

Os estudos de $\mathrm{KNOBF}^{8}$; McCORKLE \& YOUNG $^{12}$; GLINDSEY ${ }^{15}$ e PIPER et al. ${ }^{16}$, também encontraram os sintomas de ansiedade, depressão, confusão mental associados com o câncer.

Segundo CULL et al. ${ }^{3}$ a dificuldade de memória e comprometimento da concentração são comuns entre os pacientes com câncer e este aspecto é de suma importância dentro da reabilitação do paciente, pois afeta diretamente a qualidade de vida desses pacientes.

No campo dos sintomas específicos de mal-estar detectamos 6 pacientes $(54,5 \%)$ com rigidez nos ombros; $4(36,4 \%)$ relatos de falta de ar, sede, voz rouca; $2(18,2 \%)$ relataram dor de cabeça, tontura; $1(9,1 \%)$ tremores nas pálpebras e nos ombros. De acordo com SMETS et al. ${ }^{23}$ os mecanismos que produzem a fadiga são desconhecidos; estudos tem relacionado a fadiga com as conseqüências da doença que dependem do tratamento e do momento do tratamento. Alguns mecanismos somáticos tem sido relacionados com a fadiga como a desnutrição provocada pela anorexia, mudanças de metabolismo, vômitos, diarréia; a anemia é um outro fator que pode levar ao cansaço durante o dia.

Ao indagarmos a que os pacientes atribuíam esses sintomas de cansaço identificamos $4(36,4 \%)$ relatos de preocupação com a doença; 2 (18,2\%) atribuíram a ansiedade, rotinas diárias, radioterapia e cirurgia; $1(9,1 \%)$ relacionou ao estresse, trabalho, depressão e tensão emocional.

HAYES $^{6}$ ao discutir a etiologia da fadiga, mencionou a possibilidade da influência dos fatores psicológicos como a depressão devido a preocupação com a fatalidade da doença; ressalta ainda, que a depressão pode não ser causa da fadiga e sim o resultado do sentimento constante do cansaço e conclui que nos pacientes com câncer, a depressão e fadiga podem ocorrer concomitantemente, ambos como resultados de fatores biológicos.
Ao confrontarmos a literatura com os dados encontrados, podemos inferir que os sintomas de fadiga estão relacionados com fatores psicológicos, bem como com o tratamento e a fase da doença.

Os estudos de RHODES et al. ${ }^{18}$; SMALL \& GRAYDON $^{22}$ também demonstraram que muitos pacientes com doenças crônicas incluindo o câncer apresentavam a fadiga como um sintoma.

Quanto aos métodos utilizados para aliviar esses sintomas, 7 pacientes $(63,6 \%)$ relataram que andavam e/ ou realizavam exercícios; $4(36,4 \%)$ utilizavam técnicas de relaxamento; 3 (27,3\%) alimentavam-se bem; 2 $(18,2 \%)$ repousavam, dormiam e sentavam e/ou deitavam; $1(9,1 \%)$ lia, distraía-se e trabalhava.

Os trabalhos de POTEMPA ${ }^{17}$ e SKALLA \& $\mathrm{LACASSE}^{21}$, citam como intervenções para aliviar a fadiga o descanso em pequenos períodos; sonecas, desde que não atrapalhem o sono noturno; planejamento das atividades diárias para controlar o gasto de energia; caminhadas e exercícios aeróbicos que controlam o estresse; dietas balanceadas; leituras e atividades de distração como trabalho, ouvir música, yoga e relaxamento. Os métodos utilizados citados em nosso estudo confirmam os dados descritos na literatura ${ }^{17,21}$.

\section{CONSIDERAÇÕES FINAIS}

Este estudo demonstrou que a fadiga é um sintoma comum entre os pacientes laringectomizados. A literatura relaciona a fadiga com fatores psicológicos, tratamento e a fase da doença. Também verificamos essa relação em nossa pesquisa, entretanto, estudos mais aprofundados deverão ser realizados.

Os sintomas da fadiga entre os pacientes com câncer são de grande importância dentro de sua reabilitação, pois afetam diretamente a qualidade de vida dos mesmos. Portanto devemos reconhecê-los e orientar aos pacientes sobre a utilização de mecanismos para aliviar esses sintomas, tais como: caminhar, praticar exercícios, alimentação balanceada, ler, atividades de lazer, distrações entre outros já citados e praticados por alguns pacientes estudados.

\section{FATIGUE IN PATIENTS WITH LARYNGEAL CANCER}

This study searched for subjective elements in the fatigue of laryngectomized patients. The authors used an instrument proposed by YOSHITAKE ${ }^{27}$ that investigates the symptoms of physical indisposition, mental weariness and a specific sensation of fatigue. Data were analysed considering the literature, demonstrating that the psychological factors, the treatment and phases of the disease are directly related. 


\section{LA FATIGA EN PACIENTES CON CÁNCER DE LARINGE}

Este estudio observó la presencia de sintomas subjetivos de fatiga en pacientes laringectomizados. Utilizamos el instrumento propuesto por YOSHITAKE ${ }^{27}$ que investiga los sintomas de malestar, cansancio mental y sensaciones especificas de cansancio. Los datos fueron confrontados con la literatura, demostrando que los factores psicológicos, el tratamiento y la fase de la enfermedad están directamente relacionadas.

TÉRMINOS CLAVES: fatiga, pacientes, neoplasmas laringeos

\section{ANEXO 1}

\section{Identificação}

Iniciais do nome:

Sexo:

Idade:

Data do início da doença:

Data da cirurgia:

Tratamento com radioterapia ( ) Sim （ ) Não

Há quanto tempo?

Tratamento com quimioterapia ( ) Sim ( ) Não

Há quanto tempo ?

Faz uso de medicamento ? ( ) Sim( ) Não

Quais medicamentos?

I - Lista de Sintomas de Mal-Estar ${ }^{26}$

Sintomas Gerais de Mal-Estar

1. Sensação de peso na cabeça

2. Sensação de cansaço no corpo todo

3. Sensação de cansaço nas pernas

4. Bocejo

5. Sensação de cérebro quente

6. Torpor

7. Cansaço nos olhos

8. Movimentos tornam-se duros e desajeitados

9. Sensação de instabilidade ao ficar parado

10. Desejo de se deitar

Cansaço Mental

11. Sensação de dificuldade em pensar

12. Cansaço ao falar

13. Nervosismo

14. Incapacidade de concentração

15. Incapacidade de se interessar em pensamento

16. Esquecer-se com facilidade

17. Falta de autoconfiança

18. Ansiedade constante

19. Incapacidade de ficar ereto
20. Sem paciência

Sintomas Especificos de Mal-Estar

21. Dor de cabeça

22. Rigidez nos ombros

23. Dor na barriga

24. Falta de ar

25. Sede

26. Voz rouca

27. Tontura

28. Tremores nas pálpebras

29. Tremores nos membros

30. Sensação de estar doente

II - A que você atribui esses sintomas de cansaço ?

( ) Estresse

( ) Preocupação

( ) Depressão

( ) Ansiedade

( ) Tensão emocional

( ) Trabalho

( ) Rotinas diárias

( ) Radioterapia

( ) Cirurgia

( ) Quimioterapia

( ) Outros. Especificar:

III - Quais os métodos que você utiliza para aliviar esses sintomas de cansaço ?

( ) Repouso

( ) Sono

( ) Outras atividades

( ) Sentar-se / Deitar-se

( ) Leitura

( ) Andar/Exercícios

( ) Distração

( ) Relaxamento

( ) Alimentação

( ) Outros. Especificar :

\section{REFERÊNCIAS BIBLIOGRÁFICAS}

01. BRASIL. Ministério da Saúde. Secretaria Nacional de Assistência à Saúde. Instituto Nacional de Câncer. Coordenação de Programas de Controle de Câncer - Pró-Onco. Estimativa da incidência e mortalidade por câncer no Brasil. Rio de Janeiro: Pró-Onco, 1996. p.19.

02. CAMERON, C. Theory of fatigue. Ergonomics, v. 16, n. 5, p. 633-648, 1973.

03. CULL, A. et al. What do cancer patients mean they complain of concentration and memory problems? Br.J.Cancer, v. 74, p. 167-169, 1996.

04. DAVIS, C.A. Interferon-induced fatigue. Oncol. Nurs. Forum Suppl., v. 11, n. 2, p. 67, 1984.

05. FERREIRA, A.B.de H. Novo dicionário da língua portuguesa. 2. ed. Rio de Janeiro: Nova Fronteira, 1986.

06. HAYES, J.R. Depression and chronic fatigue in cancer patients. Primary Care, v. 18, p. 327-339, 1991. 
07. HAYLOCK, P.; HART, L. Fatigue in patients receiving localized radiation. Cancer Nurs., v. 2, p. 461-467, 1979.

08. KNOBF, M. Physical and psychological distress associated with adjuvant chemotherapy in women with breast cancer. J.Clin.Oncol., v. 4, p. 678684, 1996.

09. LINO, B.H. A fadiga. In: CONGRESSO NACIONAL DE PREVENÇÃO DE ACIDENTES DE TRABALHO, 17. Anais. São Paulo: FUNDACENTRO, 1978. p. 101-114.

10. MARZIALE, M.H.P. Estudo da fadiga mental de enfermeiros atuantes em instituição hospitalar com esquema de trabalho em turnos alternantes. Ribeirão Preto, 1990. p.122. Dissertação (Mestrado) - Faculdade de Filosofia Ciências e Letras de Ribeirão Preto, Universidade de São Paulo.

11. MAYO, E. Problemas humanos de una civilización industrial. Coleccion el hombre, la sociedad y la história. Buenos Aires, 1959.

12. McCORKLE, R.; YOUNG, K. Development of a symptom distress scale. Cancer Nurs., v. 1, p. 373-378, 1978.

13. NAIL, L.M. Coping with intracavitary radiation treatment for gynecologic cancer. Cancer Practice, v. 1, p. 218-224, 1993.

14. PIPER, B.F. Fatigue. In: CARRIERI, V.K.; LINDSEY, A.M.; WEST, C.W. (eds.) Pathophysiological phenomena in nursing: human responses to illness. Philadelphia: W.B. Saunders,1986. p. 219234.

15. PIPER, B.F.; LINDSEY, A.M. Measurement issues related to fatigue in cancer patients. Oncol. Nurs. Forum Suppl., v. 14, n. 2, p. 129, 1987.
16. PIPER, B. et al. Recent advances in the management of biotherapy-related side effects: fatigue. Oncol. Nurs. Forum., v. 16, n. 6, p. 27-34, 1989.

17. POTEMPA, K.M. Chronic fatigue. Annu.Rev.Nurs.Res., v. 11, p. 57-76, 1993.

18. RHODES, V.; WATSON, P.; HANSON, B. Patients' descriptions of the influence of tiredness and weakness on self-care abilities. Cancer Nurs., v. 11, p. 186-194, 1988.

19. RYAN, A.H. Varieties of fatigue. Am.J.Psychol., v. 57, p. 565-569, 1994.

20. SAITO, Y.; KOGI, K.; KASHIWAGI, S. Factors underlying subjective feelings of fatigue. J.Science Labour, v. 46, n. 4, p. 205-224, 1970.

21. SKALLA, K.A.; LACASSE, C. Fatigue and the patient with cancer: what is it and what can I do about it ?. Oncol.Nurs.Forum, v. 19, n. 10, p. 1540-1541, 1992.

22. SMALL, S.; GRAYDON, J. Perceived uncertainly, physical symptoms, and negative mood in hospitalized patients with chronic obstructive pulmonary disease. Heart Lung, v. 21, n. 6, p. 568-574, 1992.

23. SMETS, E.M.A. et al. Fatigue in cancer patients. Br.J.Cancer, v. 68, p. 220-224, 1993.

24. VOLLE, M.; BRISSON, G.R.; DION, M.; TANAKA, $M$. Travail fatigue et fréquence de fusion critique usuelle. Ergonomics, v. 21, n. 7, p. 551-558, 1978.

25. YOSHITAKE, $H$. Rating the feelings of the fatigue. J.Science Labour, v. 45, n. 7, p. 422-432, 1969.

26. YOSHITAKE, H. There are characteristic patterns of subjective fatigue symptoms. Ergonomics, v. 21, n. 3, p. 231-233, 1978.

27.YOSHITAKE, H. Relations between the symptoms and feeling of fatigue. Ergonomics, v. 14, n. 1, p. 175-186, 1971. 